\title{
THE INVALIDITY OF THE CALDERON-ZYGMUND INEQUALITY FOR SINGULAR INTEGRALS OVER LOCAL FIELDS
}

BY JAMES E. DALY

Communicated March 20, 1975

We will show that the Calderón-Zygmund inequality, $\left\|T_{\omega}\right\|_{p} \leqslant C(p, r)\|\omega\|_{r}$, is not valid in the local field setting. A complete proof of the validity of this inequality in the case of singular integrals over $\mathbf{R}^{n}$ can be found in Dunford and Schwartz, Linear operators, Vol. 2. We use the theory of regular functions as developed by $M$. Taibleson [4] and the F. and M. Riesz theorem for local fields as proved by J. Chao [1].

We assume the reader is familiar with elementary local field analysis and singular integrals in general. In the following work $K$ will denote a local field (nondiscrete, zero-dimensional, locally compact field), $B^{n}=\left\{x \in K:|x| \leqslant q^{-n}\right\}, D^{n}=$ $\left\{x \in K:|x|=q^{-n}\right\}$, and $\xi_{A}$ the characteristic function of the set $A$. Haar measure $\lambda$ is normalized so that $\lambda\left(B^{0}\right)=1 \quad\left(\lambda\left(B^{1}\right)=q^{-1}\right)$ and the prime $\pi$ is chosen so that $\pi B^{0}=B^{1}$. The fundamental character $\chi$ is trivial on $B^{0}$ and nontrivial on $B^{-1} . C_{00}$ and $C_{0}$ denote the continuous functions with compact support and the continuous functions that vanish at infinity, respectively.

Definition. For $x \in k, k \in \mathbf{Z}$, let

$$
f(x,-k)=\left\{\begin{array}{l}
0, \quad k<2, \\
\xi_{D^{0}}(x) \Sigma_{j=2}^{k} \chi\left(\pi^{-j} x\right) \quad \text { if } k \geqslant 2 .
\end{array}\right.
$$

LEMMA 1. The function $f$ defined above is regular.

Proof. A function $g: K \times \mathbf{Z} \rightarrow \mathbf{C}$ is said to be regular if

$$
g(x, k)=q^{-k} \int_{B^{-k}} g(y-x, k-1) d y .
$$

A straightforward calculation shows that $f$ satisfies this equality.

LEMMA 2.

(a) $\quad \hat{f}(x,-k)=\frac{q-1}{q} \sum_{j=2}^{k} \xi_{\pi-j+B^{0}}(x)-\frac{1}{q} \sum_{j=2}^{k} \xi_{\pi-j+D^{-1}}(x)$.

(b) $\quad\|f(\cdot,-k)\|_{2}=\{(q-1)(k-1) / q\}^{1 / 2}$ for $k \geqslant 2$.

(c) $\|f(\cdot,-k)\|_{r} \leqslant\{(q-1)(k-1) / q\}^{(r-1) / r}$ for $k \geqslant 2, \quad 2<r<\infty$.

AMS (MOS) subject classifications (1970). Primary 43A85, 44A25, 47 A30.

Key words and phrases. Singular integral, local field, Calderón-Zygmund inequality. 
Proof.

$$
\begin{aligned}
\hat{f}(x,-k) & =\int_{D^{0}} f(y,-k) \overline{\chi(x y)} d y \\
& =\int_{B^{0}} \sum_{j=2}^{k} \chi\left(\left(\pi^{-j}-x\right) y\right) d y-\int_{B^{1}} \sum_{j=2}^{k} \chi\left(\left(\pi^{-j}-x\right) y\right) d y
\end{aligned}
$$

(a)

$$
\begin{aligned}
& =\sum_{j=2}^{k} \xi_{\pi^{-j+B}}(x)-\frac{1}{q} \sum_{j=2}^{k} \xi_{\pi^{-j+B^{-1}}}(x) \\
& =\frac{q-1}{q} \sum_{j=2}^{k} \xi_{\pi^{-j+B} 0^{0}}(x)-\frac{1}{q} \sum_{j=2}^{k} \xi_{\pi^{-j}+D^{-1}}(x) .
\end{aligned}
$$

$$
\|f(\cdot,-k)\|_{2}=\|\hat{f}(\cdot,-k)\|_{2}=\{(q-1)(k-1) / q\}^{1 / 2} \quad \text { by }(\mathrm{a})
$$

(c) Let $2<r<\infty$. As $f(\cdot,-k), \hat{f}(\cdot,-k) \in C_{00},\|f(\cdot,-k)\|_{r} \leqslant\|\hat{f}(\cdot,-k)\|_{r^{\prime}}$ where $r^{\prime}=r /(r-1)$. By (a), $\|\hat{f}(\cdot,-k)\|_{r^{\prime}}=\{(q-1)(k-1) / q\}^{(r-1) / r}$.

Definition. For $k \geqslant 2$, let $\Gamma_{k}$ denote the set of $\omega: K^{*} \rightarrow \mathrm{C}$ such that

(i) $\omega\left(x+B^{k}\right)=\omega(x)$ for $x \in D^{0}$

(ii) $\omega\left(\pi^{j} s\right)=\omega(x)$ for $x \in K^{*}, j \in Z$,

(iii) $\int_{D^{0}} \omega(x) d x=0$,

and $\Gamma=\bigcup_{k=2}^{\infty} \Gamma_{k}$.

We note each $\omega \in \Gamma$ is the kernel of a singular integral operator $T_{\omega}$ (see [3]). These kernels correspond to $C^{\infty}$ kernels in the real case. We denote the multiplier of the operator $T_{\omega}$ by $F\left(T_{\omega}\right)$ and $L_{p}$-operator norm of $T_{\omega}$ by $\left\|T_{\omega}\right\|_{p}$. By $\|\omega\|_{r}$, we mean $\left\{\int_{D^{0}}|\omega(x)|^{r} d x\right\}^{1 / r}$ if $1 \leqslant r<\infty$ and $\sup _{x \in D^{0}}|\omega(x)|$ if $r=\infty$.

LEMmA 3. If $\omega \in \Gamma_{k}$, then

$$
F\left(T_{\omega}\right)(y)=\int_{D^{0}} \omega(x) \sum_{j=1}^{k} \frac{.}{\chi\left(\pi^{-j} x y\right)} d x \quad \text { for } y \in D^{0} .
$$

Consequently $F\left(T_{\omega}\right)$ is constant upon the cosets of $B^{k}$ in $D^{0}$.

Proof. See [2, Proposition 1 and Corollary 2].

For the next theorem we preclude the case of even $q$. The F. and M. Riesz theorem as proved by $\mathrm{J}$. Chao requires this restriction.

THEOREM 1 . For $1<p<\infty, 1 \leqslant r \leqslant \infty$, there is no constant $C(p, r)$ such that for all $\omega \in \Gamma$,

$$
\left\|T_{\omega}\right\|_{p} \leqslant C(p, r)\|\omega\|_{r} .
$$

Proof. From the inequalitites $\left\|T_{\omega}\right\|_{2} \leqslant\left\|T_{\omega}\right\|_{p}$ and $\|\omega\|_{r} \leqslant\|\omega\|_{\infty}$, we need only show there is no constant $C$ such that $\left\|T_{\omega}\right\|_{2} \leqslant C\|\omega\|_{\infty}$ for all $\omega \in \Gamma$. We will accomplish this if we find a sequence $\left\{\omega_{k}\right\} \subset \Gamma$ such that $\left\|\omega_{k}\right\|_{\infty} \leqslant 2$ and $\left\|T_{\omega_{k}}\right\|_{2}=\left\|F\left(T_{\omega_{k}}\right)\right\|_{\infty} \geqslant\left|F\left(T_{\omega_{k}}\right)(1)\right| \rightarrow \infty$. To this end we define for $x \in D^{0}$, 


$$
g(x,-k)= \begin{cases}f(x,-k) /|f(x,-k)| & \text { if } f(x,-k) \neq 0 \\ 0 & \text { if } f(x,-k)=0\end{cases}
$$

and

$$
\omega_{k}=g(x,-k)-\frac{q}{q-1} \int_{D 0} g(x,-k) d x .
$$

By the above definition, $\int_{D^{0}} \omega_{k}(x) d x=0$ and $\left\|\omega_{k}\right\|_{\infty} \leqslant 2$. Thus if we extend $\omega_{k}$ to $K^{*}$ by homogeneity, $\omega_{k} \in \Gamma_{k}$. We have

$$
\begin{aligned}
F\left(T_{\omega_{k}}\right)(1) & =\int_{D^{0}} \omega_{k}(x) \sum_{j=1}^{k} \overline{\chi\left(\pi^{-j} x\right)} d x \\
& =\int_{D^{0}}\left|\sum_{j=2}^{k} \chi\left(\pi^{-j} x\right)\right| d x+O(1)=\|f(\cdot,-k)\|_{1}+O(1) .
\end{aligned}
$$

By Lemma 1 the function $f$ is regular. Thus if $\|f(\cdot,-k)\|_{1} \leqslant A<\infty$, then $f$ is the regularization of a finite Borel measure $\mu$ [4, Theorem 8]. From Lemma 2(a) and the fact that $f(\cdot,-k) \rightarrow \mu$ in the weak*-topology of the dual of $C_{00}$,

$$
\hat{\mu}=\frac{q-1}{q} \sum_{j=2}^{\infty} \xi_{\pi-j+B^{0}}-\frac{1}{q} \sum_{j=2}^{\infty} \xi_{\pi-j+D^{-1}} .
$$

Thus $\hat{\mu}$ is supported on the cone $\Sigma_{j=-\infty}^{\infty} \pi^{j}\left(1+B^{1}\right)$ and, therefore, $\mu$ is absolutely continuous with respect to Haar measure [1, Corollary 5.3]. So $\mu$ is given by an $L_{1}$-function $h$ and $\hat{h}=\hat{\mu}$. But $\hat{\mu}$ is not in $C_{0}$, so $h$ cannot be in $L_{1}$. This contradiction gives $\left|F\left(T_{\omega}\right)(1)\right| \rightarrow \infty$.

We now give a variation of Theorem 1 by excluding the case $r=\infty$ in (1) and allowing $K$ to be any local field (no restriction on $q$ ). The proof is greatly simplified and depends only on Lemma 2 .

THEOREM 2. For $1<p<\infty, 1 \leqslant r<\infty$, there is no constant $C(p, r)$ such that for all $\omega \in \Gamma,\left\|T_{\omega}\right\|_{p} \leqslant C(p, r)\|\omega\|_{r}$.

Proof. As in the proof of Theorem 1, we may restrict ourselves to $\left\|T_{\omega}\right\|_{2}$. Let $\beta_{k}(x)=f(x,-k)$ for $x \in D^{0}$ and extend $\beta_{k}$ to $K^{*}$ by homogeneity. Observe $\beta_{k} \in \Gamma_{k}$. We have

$$
\begin{aligned}
F\left(T_{\beta_{k}}\right)(1) & =\int_{D^{0}} \beta_{k}(x) \sum_{j=1}^{k} \overline{\chi\left(\pi^{-j} x\right)} d x \\
& =\int_{D^{0}}\left|\sum_{j=2}^{k} \chi\left(\pi^{-j} x\right)\right|^{2} d x+\int_{D^{0}} \sum_{j=2}^{k} \chi\left(\left(\pi^{-j}-\pi^{-1}\right) x\right) d x \\
& =\|f(\cdot,-k)\|_{2}^{2}=(q-1)(k-1) / q
\end{aligned}
$$

by Lemma 2(b). Thus $\left\|T_{\beta_{k}}\right\|_{2} /\left\|\beta_{k}\right\|_{r} \rightarrow \infty$ by Lemma 2 (c). For $2 \leqslant r<\infty$, this rate of growth is greater than $\{(q-1)(k-1) / q\}^{1 / r}$.

Theorem 2 is valid in the $n$-dimensional case with no changes except for some constants in the proof. The proof of Theorem 1 can be used except one must find another method to show $\|f(\cdot,-k)\| \rightarrow \infty$, as the work of Chao is for 
dimension 1. One would expect $\|f(\cdot,-k)\|_{1}=o(\log k)$ as in the case of the classical Dirichlet kernel. A direct computation in the case $k$ is the 2 -series field substantiates this conjecture.

\section{REFERENCES}

1. J. Chao, $H^{p}$-spaces of conjugate systems on local fields, Dissertation, Washington University, 1972.

2. J. Daly, Algebras of special singular integrals over local fields, Math. Ann. (to appear).

3. K. Phillips and M. Taibleson, Singular integrals in several variables over a local field, Pacific J. Math. 30 (1969), 209-231. MR 40 \#7886.

4. M. Taibleson, Harmonic analysis on n-dimensional vector spaces over local fields. II. Generalized Gauss kernels and the Littlewood-Paley function, Math. Ann. 186 (1970), 1-19. MR 41 \#8989.

DEPARTMENT OF MATHEMATICAL SCIENCES, NEW MEXICO STATE UNIVERSITY, LAS CRUCES, NEW MEXICO 88003 